\title{
A!
}

This is an electronic reprint of the original article.

This reprint may differ from the original in pagination and typographic detail.

Al-Tous, Hanan; Tirkkonen, Olav

\section{Static Reflecting Surface Based on Population-level Optimization}

Published in:

2021 IEEE Global Communications Conference (GLOBECOM)

DOI:

10.1109/GLOBECOM46510.2021.9685836

Published: 01/02/2022

Document Version

Peer reviewed version

Please cite the original version:

Al-Tous, H., \& Tirkkonen, O. (2022). Static Reflecting Surface Based on Population-level Optimization. In 2021 IEEE Global Communications Conference (GLOBECOM) IEEE.

https://doi.org/10.1109/GLOBECOM46510.2021.9685836

This material is protected by copyright and other intellectual property rights, and duplication or sale of all or part of any of the repository collections is not permitted, except that material may be duplicated by you for your research use or educational purposes in electronic or print form. You must obtain permission for any other use. Electronic or print copies may not be offered, whether for sale or otherwise to anyone who is not an authorised user. 


\title{
Static Reflecting Surface Based on Population-level Optimization
}

\author{
Hanan Al-Tous and Olav Tirkkonen \\ Department of Communications and Networking, Aalto University, Finland \\ Email:\{hanan.al-tous, olav.tirkkonen\}@ aalto.fi
}

\begin{abstract}
We consider a Static Reflecting Surface (SRS) assisted communication system. Part of a cell served by a Base Station (BS) is blocked from Line-of-Sight (LoS), and an SRS is deployed to assist communication in that target area. The SRS has a high number of reflecting elements, with a static phase shift matrix, optimized offline at installation phase for a user population. To find the beamformer at the BS and phase shift matrix at the SRS, we formulate an optimization problem aiming to maximize the average data rate in the target area assuming LoS communication between SRS and BS, as well as between SRS and the users. A local optimum of the population-level problem is obtained using the interior point method. We furthermore consider a low complexity approach, where we divide the SRS \& BS antennas into sub-blocks and the target area into subareas; each sub-block is designed to serve a user at the center of the corresponding subarea. Simulation results show that as compared to a fully dynamic Reconfigurable Intelligent Surface (RIS) of the same size, where there is real-time electronic control of the phase shifter, an SRS loses $30 \%$ in performance. Comparing to other SRS approaches, and broadcast approach from the literature, the population based approach provides higher average Spectrum Efficiency (SE), 5\% SE and fairness index.
\end{abstract}

Index Terms-Reconfigurable Intelligent Surfaces, phase shift matrix, beamformer, Millimeter-Wave (mmWave) communication.

\section{INTRODUCTION}

Reconfigurable Intelligent Surfaces (RISs) are a promising technology for future communication systems. RISs are arrays of passively reflecting elements where the phase shifts of the elements can be intelligently changed for performance enhancement. An RIS relies on passive signal reflection and does not require any active radio frequency chains for signal transmission / reception. Moreover, RIS can be easily attached to or removed from different objects (e.g., walls and ceilings), hence exhibiting great flexibility in deployment [1], [2]. However, to control the phase shifts dynamically, RISs do require real-time electronic control, and a real-time communication interface to the radio access network.

RIS-assisted Millimeter-Wave (mmWave) massive MultipleInput-Multiple-Output (MIMO) systems are expected to offer important connectivity advantages, higher data rates and energy saving in Beyond 5G systems [3]. Leveraging the controllable reflection coefficients of large RIS, mmWave massive MIMO Base Stations (BS) can serve User Equipments (UEs) whose direct links from the BS are completely blocked by obstacles, such that the effective coverage of mmWave cellular networks can be enhanced using beamforming techniques. The design of RIS has been investigated in Orthogonal Frequency Division Multiplexing (OFDM), Non-Orthogonal Multiple Access (NOMA), and Orthogonal Multiple Access (OMA) contexts. Low-complexity suboptimal algorithms for finding the phase shift matrix have been developed based on alternating optimization, successive convex approximation and monotonic optimization techniques [4], [5]. Continuous and discrete phase shifts at the RIS are considered in [6], [7], respectively.

In an RIS, the reflection matrix is designed assuming a level of Channel State Information (CSI) of the served UEs at the BS and RIS. In [2], [8], perfect CSI is assumed. Acquiring perfect CSI is challenging due to the passivity of the RIS elements, and the high dimension of the cascaded MIMO channel. Recently, the sparsity of mmWave channels, using passive / active RIS architecture and using Machine Learning (ML) techniques are exploited to reduce training overheads in [9], [10]. In [11], an ML-based approach is considered to model the local propagation environment without relying on explicit channel estimation. Designing the beamformer and phase shift matrix based on CSI statistics is of great importance in high speed and short coherence time scenarios, where using the CSI statistics can reduce the feedback overhead required to design the phase shift matrix at the RIS. Statistical CSI is exploited to design RIS-assisted communication systems in [12], [13].

In most existing works, the reflection matrix is dynamically designed at the time of communication either to serve a single UE or multiple UEs. In [14], RIS assisted broadcasting is considered, assuming that either the transmitter or the receiver is in the near-field of the RIS. A free-space path loss model for RIS-assisted wireless communications is developed, and the RIS phase shift matrix is designed to broadcast the signal to a desired direction, represented by a single angle of arrival.

In this paper, we consider a unicast traffic scenario, but design the phase shifts of the surface and the related beamformers of the BS once and for all at the deployment phase. This renders the surface a Static Reflecting Surface (SRS); no realtime control or configuration of the surface is needed, i.e., the surface does not need electronic circuitry and the phase shifters of the SRS can be implemented using static metamaterial. The SRS is designed based on statistical CSI to serve an area where direct communication with the BS is blocked. SRSs are placed on facades of tall buildings to provide Line-of-Sight (LoS) communication in the target area. The phase shift matrix is 


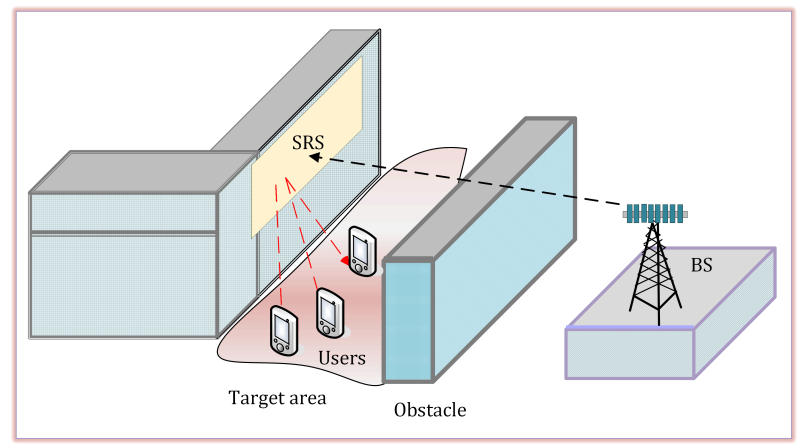

Fig. 1: System model: A population of users served by an SRSassisted communication, the direct communication of a UE with the $\mathrm{BS}$ is blocked in the target area.

designed to distribute the power uniformly in the target area. Using an SRS is attractive because of its simplicity and the possibility to be used with legacy communication systems. To the best of our knowledge, this problem has not been addressed in the literature. For a street segment facing an SRS, the azimuth and elevation direction of arrivals at the SRS span large ranges and cannot be represented by a single desired direction as in the broadcast scenario of [14].

We formulate on joint phase shift matrix and BS beamformer design problem, aiming to maximize the average rate in the target area. This problem is analytically intractable, so that we find a numerical solution based on the interior point method. To reduce computational complexity, we adopt a low complexity approach where the SRS / BS is divided into sub-blocks and the target area is divided into subareas, and each phase shifter sub-block is configured to steer the signal towards the center of the corresponding subarea.

The remainder of this paper is organized as follows. In Section II, the system model is presented. In Section III, the problem formulation and the solution approach are presented. Simulation results are presented and discussed in Section IV. Finally, conclusions are drawn in Section V.

\section{System MOdeL}

We consider a communication system with a BS and SRS. The SRS facilitates the communication of UEs in the target area as shown in Figure 1. The BS has $M=M_{v} \times M_{h}$ antenna elements, where $M_{v}$ and $M_{h}$ are the BS array elements in the vertical and horizontal dimensions, respectively. The SRS has $N=N_{v} \times N_{h}$ reflecting elements, where $N_{v}$ and $N_{h}$ are the reflecting elements in the vertical and horizontal dimensions, respectively. The UE has a single antenna. We assume that the direct links between the BS and UEs are blocked in the target area. To assist the communication of the UEs in the target area, an SRS is deployed on a building and establishes UEs communication with the BS. Assuming the SRS is deployed on the wall of a tall building, it can create channels dominated by LoS propagation along with a few scatterers. The locations of BS, SRS, and a sample UE are represented using the 3D Cartesian coordinate system as $\mathbf{b}=\left[x_{b}, y_{b}, z_{b}\right]^{T}, \mathbf{r}=\left[x_{r}, y_{r}, z_{r}\right]^{T}$ and $\mathbf{u}=\left[x_{u}, y_{u}, z_{u}\right]^{T}$, respectively. The target area $\mathcal{A}$ is specified by as

$$
\mathbf{u}_{1: 2} \in \mathcal{A}=\left\{x_{\min } \leq x_{u} \leq x_{\max }, y_{\min } \leq y_{u} \leq y_{\max },\right\},
$$

where $\left[x_{\min }, x_{\max }\right]$ and $\left[y_{\min }, y_{\max }\right]$ denote the range along the $x$-axis and $y$-axis, respectively. We assume that the UEs in the target area are at a fixed height $z_{u}=z_{0} \mathrm{~m}$. We model the channel between UE and SRS and between SRS and BS as Rician; the channel between the SRS and a UE in the target area is expressed as [13]:

$$
\mathbf{h}_{r, u}=\sqrt{L_{r, u}}\left(\sqrt{\frac{\kappa}{1+\kappa}} \mathbf{h}_{r, u}^{(L)}+\sqrt{\frac{1}{1+\kappa}} \mathbf{h}_{r, u}^{(N L)}\right),
$$

where $\mathbf{h}_{r, u} \in \mathbb{C}^{N \times 1}, L_{r, u}=\frac{\rho_{0}}{d_{r, u}^{\alpha}}$ is the path loss with $\rho_{0}$ is the path loss at the reference distance of $1 \mathrm{~m}, \alpha$ is the path loss exponent, $d_{r, u}$ is the distance between UE $\mathrm{u}$ and the SRS, i.e., $d_{r, u}=\|\mathbf{u}-\mathbf{r}\|, \kappa$ is the Rician fading factor of the SRSuser link, $\mathbf{h}_{r, u}^{(L)}$ and $\mathbf{h}_{r, u}^{(N L)}$ are the LoS and Non-LoS (NLoS) components of the channel, respectively. The LoS component is deterministic and modeled as $\mathbf{h}_{r, u}^{(L)}=\mathbf{a}_{N}\left(\theta_{r, u}, \phi_{r, u}\right)$, where the array response is expressed as:

$$
\begin{aligned}
& \mathbf{a}_{N}(\theta, \phi)= \\
& \quad\left[1, e^{-2 j \pi \mu_{h} \sin \theta \cos \phi}, \ldots, e^{-2 j \pi \mu_{h}\left(N_{h}-1\right) \sin \theta \cos \phi}\right]^{T} \\
& \quad \otimes\left[1, e^{-2 j \pi \mu_{v} \sin \theta \sin \phi}, \ldots, e^{-2 j \pi \mu_{v}\left(N_{v}-1\right) \sin \theta \sin \phi}\right]^{T},
\end{aligned}
$$

where $\theta \in[-\pi / 2, \pi / 2], \phi \in[0, \pi]$ are the elevation Angle of Arrival (AoA) / Angle of Departure (AoD) and azimuth AoA / AoD, respectively, $\mu_{h}$ and $\mu_{v}$ are the array elements spacing in units of wavelength in the horizontal and vertical directions, respectively. The elevation angle $\theta_{r, u}=\sin ^{-1}\left(\frac{z_{u}-z_{r}}{\|\mathbf{r}-\mathbf{u}\|}\right)$, and the azimuth angle $\phi_{r, u}=\cos ^{-1}\left(\frac{x_{u}-x_{r}}{\left\|\mathbf{r}_{1: 2}-\mathbf{u}_{1: 2}\right\|}\right)$. The elements of the NLoS component $\mathbf{h}_{r, u}^{(N L)}$ are molded by independently and identically distributed (i.i.d) complex Gaussian random variables. The channel between BS and SRS is modeled as:

$$
\mathbf{G}_{b, r}=\sqrt{L_{b, r}}\left(\sqrt{\frac{\nu}{1+\nu}} \mathbf{G}_{b, r}^{(L)}+\sqrt{\frac{1}{1+\nu}} \mathbf{G}_{b, r}^{(N L)}\right),
$$

where $\mathbf{G}_{b, r} \in \mathbb{C}^{N \times M}, L_{b, r}$ is the path loss between BS and SRS, i.e., $L_{b, r}=\frac{\rho_{0}}{d_{b, r}^{\alpha}}, d_{b, r}=\|\mathbf{b}-\mathbf{r}\|, \nu$ is the Rician factor of the BS-SRS link, and the LoS components of the BS-SRS link is modeled as $\mathbf{G}_{b, r}^{(L)}=\mathbf{a}_{N}\left(\theta^{\prime}, \phi^{\prime}\right) \mathbf{a}_{M}^{H}\left(\theta^{\prime \prime}, \phi^{\prime \prime}\right)$, where $\theta^{\prime}, \phi^{\prime}$ are the elevation and azimuth AoD towards the BS, $\theta^{\prime \prime}$, $\phi^{\prime \prime}$ the elevation and azimuth AoA at the BS from SRS. The elements of the NLoS component $\mathbf{G}_{b, r}^{(N L)}$ are modeled by i.i.d complex Gaussian random variables. With the help of SRS, the received signal (down-link communication) at UE $u$ can be written as:

$$
y_{u}=\sqrt{p} \mathbf{h}_{u}^{H} \mathbf{\Psi} \mathbf{G}_{b, r} \mathbf{w} s_{u}+n_{u},
$$

where $\boldsymbol{\Psi}=\operatorname{diag}\left(\left[e^{j \psi_{1}}, \ldots, e^{j \psi_{N}}\right]\right)$ denotes the phase shift matrix at the SRS, $e^{j \psi_{n}}$ denotes the phase coefficient at the nth element of the SRS and the amplitude coefficients of all elements are set to one, $\mathbf{w} \in \mathbb{C}^{M \times 1}$ is the beamformer vector at the BS with $\|\mathbf{w}\|=1$, and $s_{u}$ is the transmitted symbol towards UE u with $\mathbb{E}\left\{\left|s_{u}\right|^{2}\right\}=1, p$ is the BS transmitted 
power and $n_{u}$ is additive white Gaussian noise at the UE with noise variance $\sigma^{2}$. The spectral efficiency of UE $u$ in [bits/Hz] is expressed as:

$$
R_{u}=\log _{2}\left(1+\frac{p\left|\mathbf{h}_{u}^{H} \mathbf{\Psi} \mathbf{G}_{b, r} \mathbf{w}\right|^{2}}{\sigma^{2}}\right) .
$$

\section{Problem Formulation and Solution ApProach}

Since the xy-coordinates of target and the BS and SRS locations are known, the phase shift matrix and the beamformer vector can be designed based on the deterministic part of the CSI of the SRS-BS and SRS-UE links in the target area. Let $\bar{R}_{u}$ be defined based on the deterministic part of the CSI as:

$$
\bar{R}_{u} \triangleq \log _{2}\left(1+\frac{\gamma_{0}\left|\mathbf{h}_{u}^{(L) H} \mathbf{\Psi} \mathbf{G}_{b, r}^{(L)} \mathbf{w}\right|^{2}}{d_{r, u}^{\alpha} d_{b, r}^{\alpha}}\right),
$$

where $\gamma_{0}=\frac{p \nu \kappa \rho_{0}^{2}}{(1+\nu)(1+\kappa) \sigma^{2}}$.

The joint phase shift matrix and beamformer design problem in the target area is formulated as:

$$
\begin{gathered}
\max _{\mathbf{w}, \mathbf{\Psi}} \mathbb{E}_{\mathcal{A}}\left\{\bar{R}_{u}\right\}, \\
\text { subject to: } \psi_{n} \in[0,2 \pi), n=1, \ldots, N, \\
\|\mathbf{w}\|=1,
\end{gathered}
$$

where the expectation is with respect to the UE distribution on the target area $\mathcal{A}$, i.e.,

$$
\mathbb{E}_{\mathcal{A}}\left\{\bar{R}_{u}\right\}=\int_{x_{\min }}^{x_{\max }} \int_{y_{\min }}^{y_{\max }} \bar{R}_{u} f_{x y}\left(x_{u}, y_{u}\right) d x_{u} d y_{u},
$$

with $f_{x y}\left(x_{u}, y_{u}\right)$ represents the probability density function of the UE spatial distribution. In this paper, we assume that the UEs are uniformly distributed in the target area. The joint optimization problem (6) with respect to $\mathrm{w}$ and $\Psi$ is not a convex optimization problem, and it is not possible to compute the $2 \mathrm{D}$ integral in a closed form expression. However, a local optimum of the problem can be obtained based on the interior point method. Note, that the beamformer and phase shift matrix need to be configured only once based on deterministic part of CSI. There is no need to update or estimate the CSI at the SRS and BS.

To reduce the computational complexity related to the $2 \mathrm{D}$ integral in (7), a sub-optimal approach can be applied, where the target area is divided in to subareas, and a UE at the center of each subarea is used to represent it, then the $2 \mathrm{D}$ integral is converted to summation.

\section{A. Low Complexity Approach}

Assuming that the number of BS antenna $M$ and the number of SRS elements $M$ are large, we can divide the SRS and BS elements into $S$ sub-blocks, i.e., $N^{\prime}=N_{h}^{\prime} \times N_{v}^{\prime}$, $M^{\prime}=M_{h}^{\prime} \times M_{v}^{\prime}, S N^{\prime}=N$, and $S M^{\prime}=M$ and divide the target area into $S$ subareas, the centering physical location of each subarea is selected to determine a desired direction. The deterministic CSI towards the SRS / BS sub block-elements can be used to determine the sub-block phase shift and beamformer

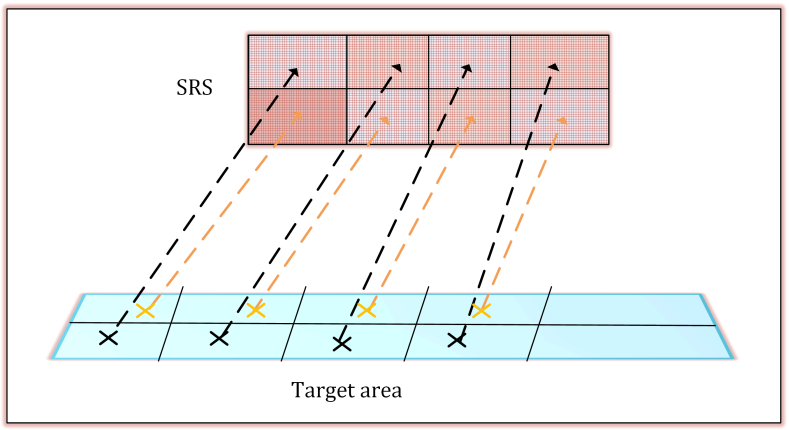

Fig. 2: The division of the SRS into $S$ sub-blocks and the target area into $S$ subareas.

matrix. Figure 2, shows the divisions of the SRS into subblocks and the target area into subareas, where the phase shift of each SRS sub-block is designed to serve a UE at the center of the corresponding subarea. Let the deterministic CSI (i.e., the LoS component) of a UE at the center of subarea $k$ towards sub-block $k$ of the SRS denoted as $\mathbf{h}_{k}^{(L)} \in \mathbb{C}^{N^{\prime} \times 1}$, for $k=1, \ldots, S$, the distance between the SRS and the center of subarea $k$ denoted by $d_{r, k}$ and the deterministic CSI between sub-block $k$ of BS antenna and sub-block $k$ of SRS denoted by $\mathbf{G}_{k}^{(L)} \in \mathbb{C}^{N^{\prime} \times M^{\prime}}$. The joint phase shift and beamformer problem for sub-block $k$ is formulated as:

$$
\begin{gathered}
\max _{\mathbf{w}_{\mathbf{k}}, \mathbf{\Psi}_{k}} \bar{R}^{(k)}, \\
\text { subject to: } \psi_{n} \in[0,2 \pi), \text { for } n=1, \ldots, N^{\prime}, \\
\left\|\mathbf{w}_{k}\right\|=1 / \sqrt{S},
\end{gathered}
$$

where $\bar{R}^{(k)}$ is the SE (based on the deterministic part of the CSI) of a UE at the center of sub-segment $k$ served by subblock $k$ of the BS and sub-block $k$ of SRS defined as:

$$
\bar{R}^{(k)} \triangleq \log _{2}\left(1+\frac{\gamma_{0}\left|\mathbf{h}_{k}^{(L) H} \mathbf{\Psi}_{k} \mathbf{G}_{k}^{(L)} \mathbf{w}_{\mathbf{k}}\right|^{2}}{d_{r, k}^{\alpha} d_{b, r}^{\alpha}}\right),
$$

where $\mathbf{w}_{k} \in \mathbb{C}^{M^{\prime} \times 1}$ represents the beamformer of BS subblock $k$ and $\boldsymbol{\Psi}_{k}$ is a diagonal phase shift matrix of SRS subblock $k$, i.e., $\boldsymbol{\Psi}_{k}=\operatorname{diag}\left(\left[e^{j \psi_{1}}, \ldots, e^{j \psi_{N}^{\prime}}\right]\right)$. Constraint (8c), is used to make sure that $\|\mathbf{w}\|=1$ with $\mathbf{w}=\left[\mathbf{w}_{1}^{T}, \ldots, \mathbf{w}_{S}^{T}\right]^{T}$, assuming uniform UE spatial distribution and that all sub blocks contain the same number of SRS / BS elements.

Problem (8) is not a convex optimization with respect to $\mathbf{w}_{k}$ and $\boldsymbol{\Psi}_{k}$. To find a sub optimal solution, we follow an iterative alternating approach as in [6]. For a given beamformer vector, the optimal phase shift can be obtained in a closed form expression. For a given phase shift matrix, the Maximum Ration Combining (MRC) is adopted to obtain the BS beamformer. The algorithm iterates between those two steps until convergence. Let $\boldsymbol{v}_{k}=\left[e^{j \psi_{1}}, \ldots, e^{j \psi_{N}^{\prime}}\right]^{T}$, $\mathbf{H}_{k}^{(L)}=\operatorname{diag}\left(\mathbf{h}_{k}^{(L) H}\right)$ and $\mathbf{f}_{k}=\mathbf{H}_{k}^{(L)} \mathbf{G}_{k}^{(L)} \mathbf{w}_{k}$, then

$$
\mathbf{h}_{k}^{(L) H} \boldsymbol{\Psi}_{k} \mathbf{G}_{k}^{(L)} \mathbf{w}_{k}=\boldsymbol{v}_{k}^{T} \mathbf{f}_{k} .
$$




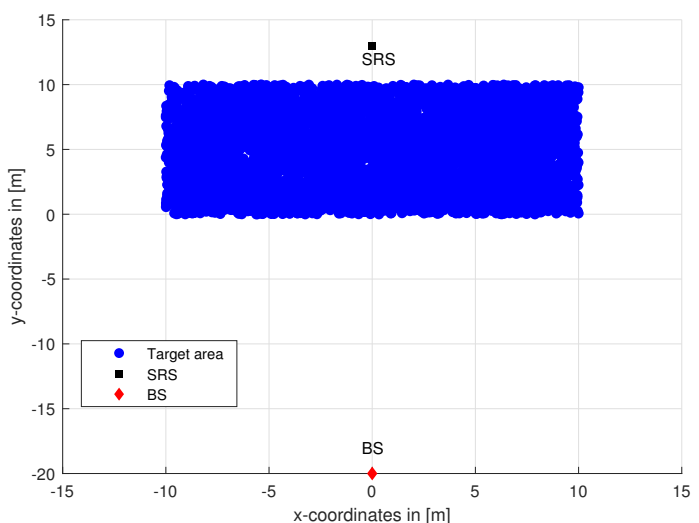

Fig. 3: Simulation layout. The target area is blue colored. The SRS location is marked by black square and the BS location is marked by red diamond.

Selecting the phase shifter for a given beamformer as

$$
\psi_{n}=-\angle \mathbf{f}_{k}(n) \text {, for } n=1, \ldots, N^{\prime},
$$

results in maximizing the average received power, where $\angle \mathbf{f}_{k}(n)$ is the angle of the nth component of the vector $\mathbf{f}_{k}$. For a given phase shift matrix $\boldsymbol{\Psi}_{k}$, the MRC beamformer at the BS is

$$
\mathbf{w}_{k}^{H}=\frac{\mathbf{h}_{k}^{(L) H} \mathbf{\Psi}_{k} \mathbf{G}_{k}^{(L)}}{\sqrt{S}\left\|\mathbf{h}_{k}^{(L) H} \mathbf{\Psi}_{k} \mathbf{G}_{k}^{(L)}\right\|} .
$$

\section{B. Practical Phase Shift Elements and BS Beamformer}

Assuming that the beamformer and reflecting coefficients are implemented using practical phase shifters. These phase shifters, however, normally have a quantized set of angles and can not shift the signal with any phase. Using $b$ bits precision, the discrete phase shift can be represented as:

$$
\psi_{n} \in\left\{0, \frac{2 \pi}{2^{b}}, \ldots, \frac{2 \pi\left(2^{b}-1\right)}{2^{b}}\right\}, \text { for } n=1, \ldots, N .
$$

The BS beamformer based on Discrete Fourier Transform (DFT) codebook is expressed as

$$
\begin{aligned}
\mathbf{c}_{p, q}= & \frac{1}{\sqrt{M}}\left[1, \ldots, e^{j 2 \pi \frac{\left(M_{h}-1\right) p}{M_{h}}}\right]^{T} \otimes\left[1, \ldots, e^{j 2 \pi \frac{\left(M_{v}-1\right) q}{M_{v}}}\right]^{T}, \\
& \text { for } p=0, \ldots, M_{h}-1, \& q=0, \ldots, M_{v}-1 .
\end{aligned}
$$

To handle practical considerations, we quantize the obtained solutions of (6) and (8). Other approaches from the literature can be adopted such as using an iterative algorithm, where on element of the phase shift is updated at each iteration, for details, see [7]. Furthermore, problem (6) / problem (8) can be solved with the added constraints (13) and (14) using the genetic algorithm as in [13].

\section{Simulations}

We consider a street segment of $10 \mathrm{~m} \times 20 \mathrm{~m}$, a BS located at $[0,-20,10]$, and an SRS located at $[0,13,15]$. The SRS facing the target area with $3 \mathrm{~m} 2 \mathrm{D}$ distance from the nearest location in the street segment. The simulation layout is shown in
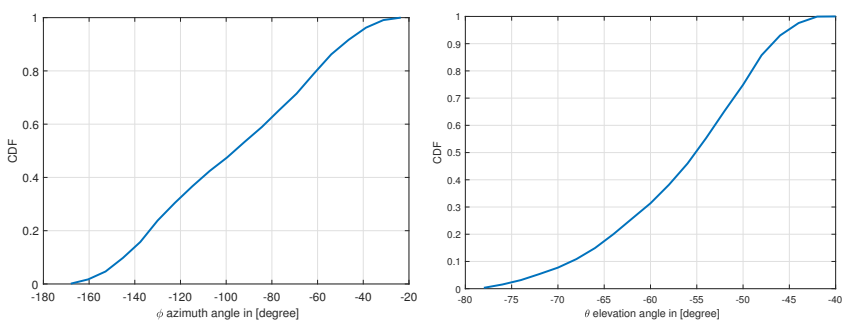

Fig. 4: The CDF of the AoA at the SRS for uniformly sampled UEs in the target area for: (Left); the azimuth AoA. (Right); the elevation AoA.

Figure 3. The BS is equipped with a Planar Linear Array (PLA) having $M=10 \times 10$ array elements with half wave-length element spacing both in the vertical and horizontal dimensions. The SRS is modeled with PLA having $N=32 \times 32$ elements with half wave-length element spacing both in the vertical and horizontal dimensions. The BS transmission power is set at 20 $\mathrm{dBm}$, the noise power is set at $-90 \mathrm{dBm}$, the center frequency is set at $28 \mathrm{GHz}$, the propagation coefficient factor is $-30 \mathrm{dBm}$, the propagation exponential is 2.2 , and the $\mathrm{k}$-factor of both UE-SRS and SRS-BS links is $9 \mathrm{~dB}$ based on the 3GPP TR 38.900 standard [15].

The Cumulative Distribution Function (CDF) of the azimuth and elevation AoAs at the SRS for 5000 UEs uniformly sampled in the street segment is shown in Figure 4. The figure shows that the AoA span is large, i.e., it covers a wide range of values. Therefore, the street segment (target area) can not be represented by a single azimuth and elevation AoAs, that can be then used to design the phase shift matrix and BS beamformer.

We compare the system performance of the following schemes:

- To benchmark the results, we consider an RIS, where the phase shift matrix and beamformer vector are dynamically optimized towards each UE location. For this scenario, the physical location of the UE is needed and the beamformer and phase shift matrix need to be updated based on the UE physical location [6]. This scenario can not be implemented using an SRS. This scenario is based on the joint active and passive beamforming approach from the literature [6], without considering the NLoS communication between BS and the UEs. This scenario is labeled by 'RIS'. For this scenario an iterative alternating approach is adopted to find the beamformer and phase shift matrix; alternating between finding the phase shift for a given beamformer and then finding the beamformer for the updated phase shift matrix, the algorithm iterates until convergence. The 'RIS' approach will provide an upper bound of the SE performance that can be achieved by other approaches.

- We consider designing the beamformer and phase shift matrix towards a UE at the center of the street segment. This scenario is labeled by 'OptCen'. This scenario is based on the broadcast scenario from the literature [14], 
with the extension of having multi antenna at the BS. The iterative alternating approach is used to find SRS phase shift matrix and the BS beamformer.

- We consider the solution of problem (6) using the interior point method, numerical integration is used to evaluate the $2 \mathrm{D}$ integral in the objective function. This scenario is labeled by 'OptPop'.

- We consider the beamformer and phase shift matrix obtained by solving problem (6), where the integration is replaced by summation of $A$ terms, where $A$ is the number of subareas, a UE is considered at the center of each subarea, and the subareas are selected to be of equal size. This scenario is labeled by 'OptSubAreas- $A$ '.

- We consider, the beamformer and phase shift matrix obtained by solving problem (8), this scenario is labeled by 'OptSubBlocks- $S$ ', where $S$ is the number of subblocks.

- We consider using random phase shift at the SRS and random beamformer at the BS. This scenario is labeled by 'Random'.

To evaluate the performance of different approaches, we consider a uniform grid in the xy-coordinates of the street segment with $0.1 \mathrm{~m}$ spacing in each dimension. The obtained phase shift matrix and beamformer for different approaches are used to compute the average SE in the target area for the UEs located in the uniform grid, using the grid make it easier to plot the results of SE as function of xy-coordinates of the street segment as discussed below.

Table I shows the average SE, 5\% SE and Jain's fairness index for different approaches/scenarios using continuous phase shifters and beamformer. Jain's fairness index is computed as $J=\frac{\left(\sum_{i=1}^{I} R_{i}\right)^{2}}{I \sum_{i=1}^{I} R_{i}^{2}}$, where $I$ is the number of UEs in the target area. The table shows that the 'RIS' approach results in the largest performance measures. The 'OptPop' approach outperforms 'OptCen' in terms of all performance measures. The 'OptSubBlocks-24' outperforms 'OptSubBlocks- $S$ ' for $S=\{6,12,48\}$ in terms of all performance measures. The perforemance of 'OptSubAreas-48 outperforms 'OptSubAreas$A^{\prime}$ for $S=\{6,12,24\}$ in terms of all performance measures. It is clear that as the number of subareas increases, the SE performance of OptSubAreas- $S$ increases. Using 'Random' approach, results in the smallest performance measures.

Table II shows the average SE, 5\% SE and Jain's fairness index for different approaches using discrete phase shift and DFT based beamformer for $b=\{2,4\}$ bits. The average SE and $5 \%$ performance measures using $b=4$ bits outperforms the SE and $5 \%$ performance measures using $b=2$ bits for 'OptPop' and 'RIS' approaches.

The CDF of the SE for different approaches is shown in Figure 5 , in the top figure, continuous phase shifts and beamformer are considered, whereas in the bottom figure discrete phase shift with different number of bits $b$ and DFT beam former are considered. The figure shows that 'OptPop' approach outperforms 'OptCen', 'OptSubAreas- $S$ ', 'OptSubBlocks- $A$ ' and 'Random' approaches.
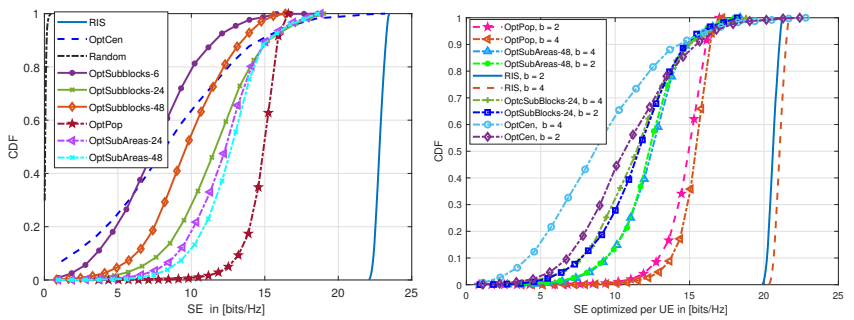

Fig. 5: The CDF of the SE for different approaches using: (Left); continuous SRS phase shifters and BS beamformer. (Right); discrete phase shifters at the SRS and DFT beamformer at the BS.
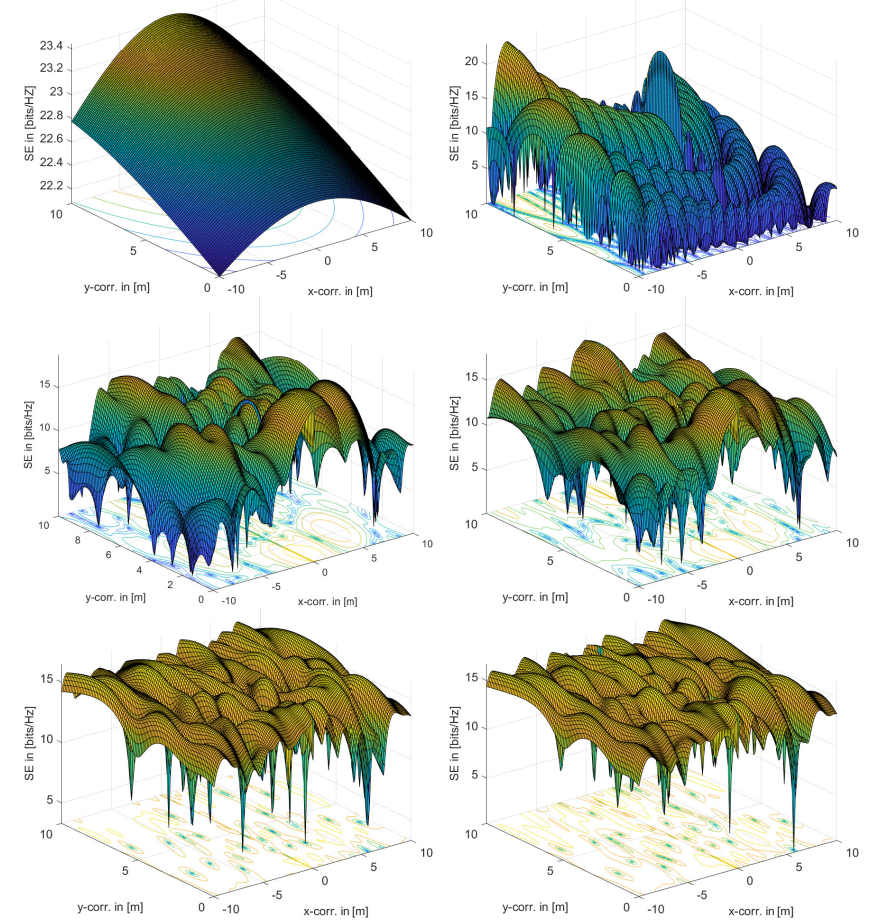

Fig. 6: The SE as a function of the $x-y$ coordinates of the target area for: (Top-Left); 'RIS' approach. (Top-Right); 'OptCen' approach. (Middle-Left); 'OptSubblocks-24' approach. (MiddleRight); 'OptSubAreas-48 approach. (Bottom-Left); 'OptPop' approach. (Bottom-Right); 'OptPop' approach with discrete phase shift for $b=2$ bits and DFT beamformer.

Figure 6 shows the SE as a function of the $\mathrm{x}-\mathrm{y}$ coordinates of the target area for different approaches. The 'RIS' approach has the best SE performance on the cost of re-configuring the phase shift and beamformer at each UE location. The 'OptPop' has a smoother SE compared to 'OptCen', 'OptSubblocks24 ' and 'OptSubAreas-48 approaches. The 'OptSubblocks24 ' has a smoother (better) SE performance compared to 'OptCen'. The 'OptSubAreas-48 has a smoother SE compared to 'OptCen' and 'OptSubblocks-24'. Using $b=2$ bits and DFT BS beamformer do not change the smooth behavior of the SE of the 'OptPop' approach.

\section{CONCLUSION}

In this paper, we considered an Static Reflecting Surface (SRS) assisted communication system. An SRS is deployed 
TABLE I: SE Performance Measures for Different Approaches

\begin{tabular}{|c|c|c|c|c|c|c|c|c|c|c|c|c|}
\hline & \multirow{2}{*}{ 'RIS' } & \multirow{2}{*}{ 'OptPop' } & \multirow{2}{*}{ 'OptCen' } & \multirow{2}{*}{ 'Random' } & \multicolumn{4}{|c|}{ 'OptSubAreas- $A^{\prime}$} & \multicolumn{4}{|c|}{ 'OptSubBlocks- $S$ ' } \\
\hline & & & & & $A=6$ & $A=12$ & $A=24$ & $A=48$ & $S=6$ & $S=12$ & $S=24$ & $S=48$ \\
\hline Average SE & 22.8 & 14.7 & 8.4 & 0.7 & 11.4 & 11.7 & 12.2 & 12.7 & 7.6 & 8.2 & 11.6 & 9.7 \\
\hline $5 \%$ SE & 22.2 & 12.5 & 0.6 & 0.004 & 7.6 & 7.9 & 8.1 & 8.8 & 2.6 & 2.7 & 6.6 & 5.0 \\
\hline Jain's Index & 0.99 & 0.99 & 0.76 & 0.51 & 0.96 & 0.96 & 0.96 & 0.97 & 0.86 & 0.85 & 0.93 & 0.92 \\
\hline
\end{tabular}

TABLE II: SE Performance Measures Using Discrete Phase Shift and DFT Beamformer for Different Approaches

\begin{tabular}{ccccccccccc}
\hline & \multicolumn{2}{c}{ 'RIS' } & \multicolumn{2}{c}{ 'OptPop' } & \multicolumn{2}{c}{ 'OptCen' } & \multicolumn{2}{c}{ 'OptSubAreas-48' } & \multicolumn{2}{c}{ 'OptSubBlocks-24' } \\
\cline { 2 - 11 } No. of bits $b$ & 2 & 4 & 2 & 4 & 2 & 4 & 2 & 4 & 2 & 4 \\
\cline { 2 - 10 } Average SE & 20.6 & 21.1 & 14.7 & 15.2 & 10.8 & 8.9 & 12.2 & 12.3 & 11.4 & 11.3 \\
5\% SE & 20.1 & 20.6 & 12.1 & 13.7 & 5.7 & 3.5 & 8.2 & 8.4 & 6.5 & 6.3 \\
Jain's Index & 0.99 & 0.99 & 0.99 & 0.99 & 0.91 & 0.84 & 0.97 & 0.97 & 0.95 & 0.94 \\
\hline
\end{tabular}

for assisting the communication between the BS and the user population in a target area, in which the direct communication with the BS is blocked. Assuming LoS communication between BS and SRS and between SRS and the target area, we formulated an optimization problem aiming to maximize the average spectrum efficiency of the users population in the target area, given CSI statistics. The optimization problem is nonconvex, and the objective function is expressed as a 2D integral. Therefore, we used the interior point method with numerical integration to find a local optimal solution of the problem. To reduce computational complexity, we devised a sub optimal algorithm to design the phase shifter and beamformer by dividing the SRS / BS into sub-blocks and the target area into subareas, and designed the phase shift and beamformer of each sub-block independently. Simulation results showed that the population based approach outperforms other approaches in terms of the average SE, 5\% SE and Jains' fairness index. The average SE of the sub-block based approach outperforms the broadcast towards a given direction from the literature. Comparing to a dynamically optimized RIS, an SRS loses some $30 \%$ in throughput. The SRS, however, needs no electronic control nor real time channel estimation between the SRS and the UEs. The SRS becomes a static part of the environment, and during operation, the BS does not need to be aware of the SRS at all. In future work, the average SE in the target area will be evaluated using CSI generated from a ray tracing model.

\section{ACKNOWLEDGMENT}

This work was funded in part by the Academy of Finland (grant 319484).

\section{REFERENCES}

[1] M. D. Renzo, M. Debbah, D. T. P. Huy, and et.al, "Smart radio environments empowered by AI reconfigurable meta-surfaces: An idea whose time has come," EURASIP J Wireless Com. Network (JWNC), vol. 129, 2019.

[2] Q. Wu and R. Zhang, "Towards smart and reconfigurable environment: Intelligent reflecting surface aided wireless network," IEEE Wireless Commun. Mag., vol. 58, no. 1, pp. 106-112, 2020.
[3] S. Hu, F. Rusek, and O. Edfors, "Beyond massive MIMO: The potential of positioning with large intelligent surfaces," IEEE Trans. Signal Process., vol. 66, no. 7, pp. 1761-1774, 2018.

[4] S. A. Busari, K. M. S. Huq, S. Mumtaz, L. Dai, and J. Rodriguez, "Millimeter-wave massive MIMO communication for future wireless systems: A survey," IEEE Commun. Surveys Tuts., vol. 20, no. 2, pp. 836-869, 2018.

[5] X. Mu, Y. Liu, L. Guo, J. Lin, and R. Schober, "Joint deployment and multiple access design for intelligent reflecting surface assisted networks," ArXiv, vol. abs/2005.11544, 2020.

[6] Q. Wu and R. Zhang, "Intelligent reflecting surface enhanced wireless network via joint active and passive beamforming," IEEE Trans. Wireless Commun., vol. 18, no. 11, pp. 5394-5409, 2019.

[7] Q. Wu and R. Zhang, "Beamforming optimization for wireless network aided by intelligent reflecting surface with discrete phase shifts," IEEE Trans. Wireless Commun., vol. 68, no. 3, pp. 1838-1851, 2020.

[8] E. Basar, M. Di Renzo, J. De Rosny, M. Debbah, M. Alouini, and R. Zhang, "Wireless communications through reconfigurable intelligent surfaces," IEEE Access, vol. 7, pp. 116753-116773, 2019.

[9] A. Taha, M. Alrabeiah, and A. Alkhateeb, "Enabling large intelligent surfaces with compressive sensing and deep learning," 2019.

[10] S. Liu, Z. Gao, J. Zhang, M. D. Renzo, and M.-S. Alouini, "Deep denoising neural network assisted compressive channel estimation for mmWave intelligent reflecting surfaces," IEEE Trans. Veh. Technol., vol. 69, no. 8, pp. 9223-9228, 2020.

[11] B. Sheen, J. Yang, X. Feng, and M. M. U. Chowdhury, "A deep learning based modeling of reconfigurable intelligent surface assisted wireless communications for phase shift configuration," IEEE Open Journal of the Communications Society, vol. 2, pp. 262-272, 2021.

[12] Y. Han, W. Tang, S. Jin, C.-K. Wen, and X. Ma, "Large intelligent surface-assisted wireless communication exploiting statistical CSI," IEEE Trans. Veh. Technol., vol. 68, no. 8, pp. 8238-8242, 2019.

[13] K. Zhi, C. Pan, H. Ren, and K. Wang, "Power scaling law analysis and phase shift optimization of RIS-aided massive MIMO systems with statistical CSI," 2021.

[14] W. Tang, M. Z. Chen, X. Chen, J. Y. Dai, Y. Han, M. Di Renzo, Y. Zeng, S. Jin, Q. Cheng, and T. J. Cui, "Wireless communications with reconfigurable intelligent surface: Path loss modeling and experimental measurement," IEEE Trans. Wireless Commun., vol. 20, no. 1, pp. 421439, 2021.

[15] 3GPP, "Study on channel model for frequency spectrum above $6 \mathrm{GHz}$," 3rd Generation Partnership Project (3GPP), Technical Report (TR) 38.900, Version 14.3.1, june 2017. 